\title{
Metabolic Changes and Diabetes Microvascular Complications 5 Years After Obesity Surgery
}

\author{
Alexander D. Miras ${ }^{1} \cdot$ Saranya Ravindra ${ }^{1} \cdot$ Anna Humphreys ${ }^{1} \cdot$ Gerassimos Lascaratos $^{1} \cdot$ Kevin N. K. Quartey $^{1}$ (D) $\cdot$ \\ Ahmed Rashid Ahmed ${ }^{2}$ - Jonathan Cousins ${ }^{2} \cdot$ Krishna Moorthy $^{2} \cdot$ Sanjay Purkayastha ${ }^{2} \cdot$ Sherif Hakky $^{2} \cdot$ Tricia Tan $^{1}$. \\ Harvinder S. Chahal ${ }^{1}$
}

Published online: 2 August 2019

(C) The Author(s) 2019

\begin{abstract}
Background Obesity surgery has pronounced effects on metabolic profile of patients with type 2 diabetes mellitus (T2DM); however, reports on long-term remission rates based on the standardised and holistic criteria by the International Diabetes Federation (IDF) and effects on T2DM microvascular complications are scarce in the literature. In this retrospective clinical trial, our objectives were to assess these variables 5 years after surgery.

Methods Clinical data and direct measurements of renal and retinal damage were collected prospectively and analysed retrospectively for 82 patients with T2DM who underwent obesity surgery and were followed up for 5 years.

Results The cohort of 82 patients with T2DM that were followed up 5 years after obesity surgery was predominantly female (71\%) with a median age of 51 years, weight of $133.5 \mathrm{~kg}$, BMI of $46.8 \mathrm{~kg} / \mathrm{m}^{2}$ and pre-operative duration of T2DM of 8 years; $6 \%$ of patients had diet-controlled T2DM, 57\% were on non-insulin treatment and 37\% were on insulin treatment pre-operatively. Of the total 82 patients, 59 patients underwent Roux-en-Y gastric bypass, 15 sleeve gastrectomy and 8 patients underwent gastric band operations. At 5 years, $5 \%$ and $15 \%$ patients achieved optimisation and improvement of the metabolic state based on the IDF criteria respectively. Surgery was associated with almost halving of the albumin-creatinine ratio in 22 patients with pre-existing albuminuria (follow-up data available for 64 patients) and an overall stabilisation of retinopathy in 24 patients with retinal images available at 5 years.

Conclusion Whilst the findings on microvascular complications are encouraging, the rates of metabolic remission were lower than expected and raise the need for validated protocols to assist clinicians in managing these patients more aggressively post-operatively to achieve optimum cardio-metabolic risk factor control and hopefully further reduction in microvascular and macrovascular complications.
\end{abstract}

Keywords Obesity $\cdot$ Diabetes $\cdot$ Long-term $\cdot$ Remission

Alexander D. Miras shared first authors.

Electronic supplementary material The online version of this article (https://doi.org/10.1007/s11695-019-04095-w) contains supplementary material, which is available to authorized users.

Kevin N. K. Quartey

kq314@ic.ac.uk; kevin.quartey14@imperial.ac.uk

Alexander D. Miras

a.miras@nhs.net

Saranya Ravindra

saranyaravindra92@gmail.com

Anna Humphreys

anna.humphreys11@imperial.ac.uk

Gerassimos Lascaratos

glascaratos@ doctors.org.uk

Ahmed Rashid Ahmed

a.ahmed07@imperial.ac.uk

\author{
Jonathan Cousins \\ jonathan.cousins1@nhs.net \\ Krishna Moorthy \\ k.moorthy@imperial.ac.uk \\ Sanjay Purkayastha \\ s.purkayastha@imperial.ac.uk \\ Sherif Hakky \\ sherif.hakky@nhs.net \\ Tricia Tan \\ t.tan@imperial.ac.uk \\ Harvinder S. Chahal \\ h.chahal@imperial.ac.uk
}

Extended author information available on the last page of the article 


\section{Introduction}

Obesity surgery has been shown to have impressive effects on the glycaemic control in obese patients with type 2 diabetes mellitus (T2DM) [1-3]; however, the definition of "diabetes remission" differs amongst the published RCTs. The American Diabetes Association (ADA) was the first to define "complete" and "partial" T2DM remission based on purely glycaemic markers [4]. The International Diabetes Federation (IDF) then introduced new more holistic criteria defining the "optimisation" and "substantial improvement" of the metabolic state using not only markers of glycaemia and medication usage but also weight loss, plasma lipids and blood pressure (BP) [5]. Evidence on 5-year T2DM "remission" rates using the IDF criteria and on the effects of the most common obesity surgery procedures on microvascular complications using direct measurements is relatively scarce in the literature (e.g. $[6,7])$.

Our primary aim was to assess the rates of T2DM patients achieving the IDF criteria 5 years after obesity surgery in order to evaluate its long-term metabolic effects. Our secondary aim was to assess the long-term effect of obesity surgery on albuminuria and retinal appearances using direct measurements.

\section{Methods}

Data were collected prospectively and analysed retrospectively for 82 obese T2DM patients who underwent obesity surgery between 2006 and 2009 at the Imperial College Healthcare NHS Trust, UK, a centre of excellence for obesity surgery. Ethical permission was obtained from the Imperial College Healthcare NHS Trust Clinical Governance and Patient Safety Committee reference: 09/808. Data were collected pre-operatively and 5 years post-operatively for clinical parameters including body weight; HbAlc; fasting blood glucose and lipids; BP; rates of reported hypoglycaemia; and number of glucose, BP and lipid-lowering medications taken. Post-operatively, these patients are managed in the specialist obesity clinic for 2 years and then routinely discharged to primary care.

Partial or complete remission of T2DM and optimisation or improvement of the metabolic state at 5 years were defined based on the IDF and ADA criteria respectively (Supplemental Table 1).

Albuminuria was assessed through the mean of two early morning spot urine albumin-creatinine ratio (ACR) values of 62 patients. This cohort was further divided into those with abnormal ACR values pre-operatively as defined by $>2.5 \mathrm{mg} /$ mmol for men and $>3.5 \mathrm{mg} / \mathrm{mmol}$ for women. Retinal appearances were assessed in 24 patients with two-field digital retinal images obtained from the English National Screening Programme for Diabetic Retinopathy and graded by an independent ophthalmologist who was blinded to the patient clinical information, using a five-stage disease severity classification for diabetic retinopathy from the International Clinical Diabetic Retinopathy and Diabetic Macular Oedema Disease Severity Scales [8]. Improvement or worsening was defined as a decrease or increase of at least two steps in the same grading system, respectively.

\section{Statistical Analysis}

Descriptive statistics are presented as absolute values, percentages or median (25th-75th quartiles). As all datasets were not normally distributed, pre-post-surgery comparisons were made using the Wilcoxon-matched pairs test. Categorical data were analysed using chi-square testing. A $p$ value $<0.05$ was considered statistically significant. GraphPad Prism 6 and Sigmaplot version 12.0 statistical software packages were used.

\section{Results}

Our cohort of 82 patients with complete paired datasets at baseline and 5 years after obesity surgery was predominantly female $(71 \%)$ with a median age of 51 years, median weight of $133.5 \mathrm{~kg}$, median BMI of $46.8 \mathrm{~kg} / \mathrm{m}^{2}$ and pre-operative duration of diabetes of 8 years; $72 \%$ underwent Roux-en- $Y$ gastric bypass (RYGB) $(n=59), 18 \%$ underwent vertical sleeve gastrectomy (VSG) $(n=15)$ and $10 \%$ underwent gastric band (BAND) $(n=8)$. Pre-operatively, $6 \%$ of patients had dietcontrolled T2DM, 57\% were on non-insulin treatment and $37 \%$ were on insulin treatment. No patients met the ADA or IDF criteria at baseline.

Pre-operative and 5-year post-operative data are shown in Table 1. BMI, HbA1c, fasting glucose, triglycerides, systolic and diastolic BP were all significantly decreased 5 years after surgery compared to baseline. There was a significant increase in HDL cholesterol. The decrease in total cholesterol and LDL cholesterol at 5 years did not achieve statistical significance. There was a significant decrease in the median number of glucose-lowering medications used at 5 years compared to baseline, but no significant change in BP and lipid-lowering medication usage. Forty-three percent of patients were on no T2DM medications, $43 \%$ on non-insulin treatment and $15 \%$ on insulin treatment 5-years post-operatively. During the 5year post-operative period, $32 \%$ of patients experienced at least one episode of hypoglycaemia.

At 5 years post-operatively, $5 \%(n=4)$ and $15 \%(n=12)$ of patients met the IDF criteria for optimisation and improvement of the metabolic state respectively (Fig. 1). Twentythree percent $(n=19)$ and $37 \%(n=30)$ of patients met the ADA criteria for complete glycaemic remission of T2DM and partial remission respectively. 
Table 1 Summary of the pre-operative and 5-year post-operative data from the cohort of patients studied $(n=82)$

\begin{tabular}{|c|c|c|c|}
\hline & Pre-operatively & 5 year post-operatively & $P$ value \\
\hline Age (years) & $51.1 \pm 10.0(28.0-70.0)$ & $\mathrm{n} / \mathrm{a}$ & $\mathrm{n} / \mathrm{a}$ \\
\hline Gender F/M $(n)$ & $58 / 24$ & $\mathrm{n} / \mathrm{a}$ & $\mathrm{n} / \mathrm{a}$ \\
\hline Weight (kg) & $133.5[113.7-150.0](84.0-235.0)$ & $100.4[82.2-115.0](62.0-165.0)$ & $<0.0001$ \\
\hline$\%$ total weight loss & $\mathrm{n} / \mathrm{a}$ & $24.0 \pm 11.8(-3.0-50.7)$ & $\mathrm{n} / \mathrm{a}$ \\
\hline BMI $\left(\mathrm{kg} / \mathrm{m}^{2}\right)$ & 46.8 [41.9-52.7] (32.1-76.7) & $35.3[30.0-40.6](24.9-60.2)$ & $<0.0001$ \\
\hline HbAlc $(\%)$ & $7.7[6.7-9.3)(5.2-12.6)$ & $6.3[5.6-7.2](5.0-9.6)$ & $<0.0001$ \\
\hline $\mathrm{HbAlc}(\mathrm{mmol} / \mathrm{mol})$ & $61.0[50.0-78.0)(33.0-114.0)$ & $45.0[38.0-55.0](31.0-81.0)$ & $<0.0001$ \\
\hline Glucose (mmol/l) & $7.1[5.3-9.4](3.0-19.4)$ & $6.0[5.0-8.0](3.1-16.0)$ & 0.0014 \\
\hline Total cholesterol (mmol/l) & $4.7[4.0-5.4](2.1-8.8)$ & $4.4[3.8-5.1](2.0-9.1)$ & 0.21 \\
\hline HDL cholesterol (mmol/l) & $1.1[0.9-1.3](0.5-2.1)$ & $1.4[1.2-1.6](0.7-2.5)$ & $<0.0001$ \\
\hline LDL cholesterol (mmol/l) & $2.6[1.9-3.6](0.4-5.2)$ & $2.3[1.8-3.0](1.0-6.2)$ & 0.16 \\
\hline Triglycerides $(\mathrm{mmol} / \mathrm{l})$ & $1.8[1.3-2.4](0.6-7.4)$ & $1.3[0.9-1.8](0.4-4.4)$ & $<0.0001$ \\
\hline Systolic BP (mmHg) & $142[130-150](103-195)$ & 128 [120-139] (104-196) & $<0.0001$ \\
\hline Diastolic BP (mmHg) & 84 [80-90] (63-114) & $76[70-80](57-110)$ & $<0.0001$ \\
\hline Number of glucose lowering medication & $2[1-2](0-4)$ & $1[0-1](0-3)$ & $<0.0001$ \\
\hline Number of BP lowering medication & $1[0-2](0-5)$ & $1[0-2](0-5)$ & 0.48 \\
\hline Number of lipid lowering medications & $1[0-1](0-3)$ & $1[0-1](0-1)$ & 0.86 \\
\hline Hypoglycaemia (\%) & $\mathrm{n} / \mathrm{a}$ & $32 \%$ & $\mathrm{n} / \mathrm{a}$ \\
\hline $\mathrm{ACR}(\mathrm{mg} / \mathrm{mmol})$ & $1.8[0.8-4.9](0.2-312.4)$ & $1.2[0.7-4.3](0.1-413.4)$ & 0.48 \\
\hline ACR in cohort with albuminuria at baseline, $n=62,(\mathrm{mg} / \mathrm{mmol})$ & $7.1[4.6-29.5](2.9-312.4)$ & $3.6[0.6-18.1](0.3-413.4)$ & 0.020 \\
\hline
\end{tabular}

Descriptive statistics are presented as absolute values, percentages, or median [interquartile range] and (range). Pre-post-comparisons were performed using Wilcoxon-matched paired tests. Glucose and lipids were assessed in the fasting state. Medication usage refers to the number of glucose, BP and lipid-lowering drugs used per day and hypoglycaemia to the percentage of patients with episodes in which capillary glucose readings of $<4.0 \mathrm{mmol} / 1$ at any stage during the 5 -year post-operative period. The cohort has been divided into those with abnormal ACR values pre-operatively as defined by $>$ $2.5 \mathrm{mg} / \mathrm{mmol}$ for men and $>3.5 \mathrm{mg} / \mathrm{mmol}$ for women

$B M I$ body mass index, $H b A l c$ glycated haemoglobin, $L D L$ low-density lipoprotein, $H D L$ high-density lipoprotein, $B P$ blood pressure, $A C R$ albumin/ creatinine ratio, $n / a$ not applicable

A pre-operative duration of $\mathrm{T} 2 \mathrm{DM}$ of less than 5 years was associated with an increased likelihood of complete remission based on the ADA criteria 5 years post-operatively. Patients

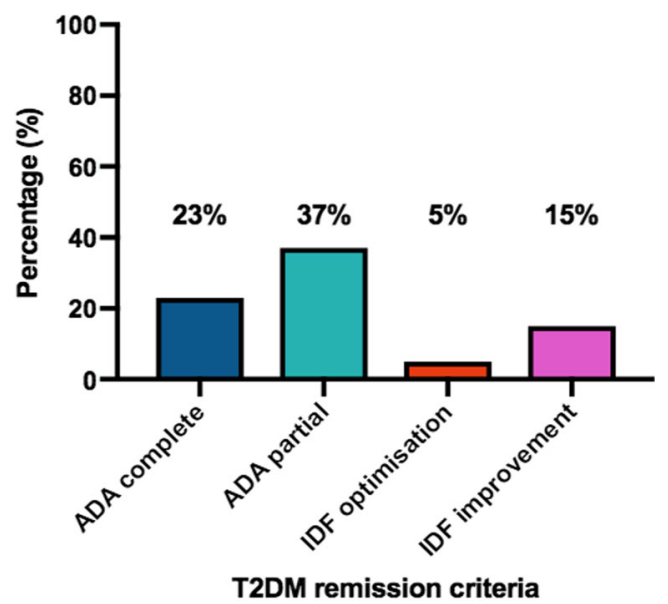

Fig. 1 Percentage of patients meeting the American Diabetes Association (ADA) criteria for complete or partial remission of type 2 diabetes mellitus (T2DM) and the International Diabetes Federation (IDF) criteria for optimisation or improvement of the metabolic state at 5 years after obesity surgery $(n=82)$ treated with insulin pre-operatively were less likely to achieve ADA complete remission compared to non-insulin treated patients. Age, gender, pre-operative BMI, HbAlc, duration of T2DM and insulin use did not significantly predict the achievement of optimisation of the metabolic state based on the IDF criteria (Supplemental Table 2). Weight loss did not predict the achievement of any of the criteria.

Amongst the surgical procedures, 5\% of RYGB patients, $0 \%$ of BAND patients and $7 \%$ of VSG patients met the IDF criteria for optimisation of the metabolic state. Twenty-five percent of RYGB patients, $25 \%$ of BAND patients and $13 \%$ of VSG patients met the ADA criteria for complete remission of T2DM. The sample size did not allow for statistical comparisons between the three groups.

In the subgroup of 62 patients ( $76 \%$ of total patient cohort) with urinary ACR, measurements were available for follow up; there was no significant change at 5 years after surgery compared to baseline (Table 1). However, there was a significant reduction in ACR in the 22 patients with albuminuria at baseline.

In the subgroup of 24 patients ( $29 \%$ of total patient cohort) with available retinal images, retinal appearances remained 
stable in 13, worsened in 6 and improved in 5 patients 5 years after obesity surgery (Supplemental Figure 1). We did not identify any factors that predicted the worsening of retinopathy.

\section{Discussion}

In this study, we have demonstrated that obesity surgery is associated with statistically significant improvements in BMI, HbAlc, fasting glucose, HDL cholesterol, triglycerides and BP even at 5 years post-operatively. However, the percentage of patients achieving the holistic IDF criteria at 5 years was lower than expected. The vast majority of available studies and RCTs report remission based on the glucocentric ADA criteria (e.g. [7]). To our knowledge, this is the first time the IDF criteria have been applied to this population. Surgery was associated with almost halving of ACR in patients with pre-existing albuminuria and an overall stabilisation of retinopathy.

Although surgery is still effective at 5 years and there is a significant improvement in many cardio-metabolic factors, $15 \%$ of patients achieved the more holistic IDF criteria for optimisation of not only glycaemia but also other cardiometabolic risk factors. The ADA criteria for complete remission were met by $23 \%$ of patients in our cohort at 5 years. This is consistent with the findings of the only other RCT that used similar criteria in which $22 \%$ of patients treated in a USA centre of excellence in bariatric surgery achieved this long-term complete remission of T2DM [7].

The relatively low remission rates reported in our study may be due to the stringency of the IDF criteria themselves or due to suboptimal long-term management of T2DM patients due to the absence of validated treatment algorithms to guide clinicians and patients. It is not uncommon practice to withdraw all or most glucose, BP and lipid-lowering medication soon after obesity surgery in anticipation of a "cure" of these comorbidities. In the UK, these patients are cared for in secondary care for 2 years before discharge to primary care. Failure to reintroduce these medications after the first 2 years, i.e. when this is usually required, may have resulted in the under-treatment of cardio-metabolic risk factors. This reluctance may be driven at least in part by medication usage as a criterion for "metabolic success" as defined both by the IDF and ADA and fear that restarting medications would suggest treatment failure. On the contrary, our findings raise the hypothesis that a synergistic approach between surgery and medical therapy may be more effective in achieving optimal control of these risk factors and may translate in better micro and macrovascular outcomes. Ultimately, meeting these targets is far more important than the number of medications used to achieve this.
Our novel and encouraging 5-year data on microvascular complication using direct measurements are in line with those published from the SOS study using indirect measurements [9] and suggest that albuminuria is more sensitive than retinopathy to the numerous physiological effects of surgery. Particular attention should be paid to those patients whose retinal appearances deteriorate and identify predictive factors.

Limitations of our study include the bias introduced by its retrospective analysis, lack of a control group and missing data on microvascular complications, which reduced our sample size. The main reason for missing data on albuminuria and retinal images was that unfortunately patients often do not attend these appointments on a regular yearly basis. Additional reasons for the rather low optimisation/remission rates were that the long average duration of T2DM before surgery and that patients were not offered procedures like the single anastomosis duodeno-ileal bypass with sleeve gastrectomy and duodenal switch.

In conclusion, although there were sustained improvements in cardio-metabolic risk factors, the proportion of patients achieving IDF criteria for optimisation of the metabolic state at 5 years following obesity surgery was lower than expected. These findings may indicate the need for validated protocols to assist clinicians in managing these patients more aggressively post-operatively to achieve optimum cardiometabolic risk factor control and reduction in microvascular and macrovascular complications.

\section{Compliance with Ethical Standards}

Conflict of Interest The authors declare that they have no conflict of interest.

Informed Consent Informed consent was obtained from all individual participants included in the study.

Ethical Approval Ethical permission was obtained from the Imperial College Healthcare NHS Trust Clinical Governance and Patient Safety Committee reference: 09/808.

Open Access This article is distributed under the terms of the Creative Commons Attribution 4.0 International License (http:// creativecommons.org/licenses/by/4.0/), which permits unrestricted use, distribution, and reproduction in any medium, provided you give appropriate credit to the original author(s) and the source, provide a link to the Creative Commons license, and indicate if changes were made.

\section{References}

1. Schauer PR, Bhatt DL, Kirwan JP, et al. Bariatric surgery versus intensive medical therapy for diabetes - 3-year outcomes. N Engl J Med. 2014;370:2002-13.

2. Mingrone G, Panunzi S, de Gaetano A, et al. Bariatric-metabolic surgery versus conventional medical treatment in obese patients with type 2 diabetes: 5 year follow-up of an open-label, single-centre, 
randomised controlled trial. Lancet. 2015;386(9997):964-73. https:// doi.org/10.1016/S0140-6736(15)00075-6.

3. Cummings DE, Arterburn DE, Westbrook EO, et al. Gastric bypass surgery vs intensive lifestyle and medical intervention for type 2 diabetes: the CROSSROADS randomised controlled trial. Diabetologia. 2016;59(5):945-53.

4. Buse JB, Caprio S, Cefalu WT, et al. How do we define cure of diabetes? Diabetes Care. 2009;32(11):2133-5.

5. Dixon JB, Zimmet P, Alberti KG, et al. Bariatric surgery: an IDF statement for obese type 2 diabetes. Diabet Med. 2011;28(6):628-42.

6. Ikramuddin S, Korner J, Lee WJ, et al. Lifestyle intervention and medical management with vs without roux-en-Y gastric bypass and control of hemoglobin A1c, LDL cholesterol, and systolic blood pressure at 5 years in the Diabetes Surgery Study. JAMA. 2018;319(3):266-78. https://doi.org/10.1001/jama.2017.20813.
7. Schauer PR, Bhatt DL, Kirwan JP, et al. Bariatric surgery versus intensive medical therapy for diabetes - 5-year outcomes. N Engl J Med. 2017;376(7):641-51.

8. Wilkinson CP, Ferris III FL, Klein RE, et al. Proposed international clinical diabetic retinopathy and diabetic macular edema disease severity scales. Ophthalmology. 2003;110(9):1677-82.

9. Sjostrom L et al. Association of bariatric surgery with long-term remission of type 2 diabetes and with microvascular and macrovascular complications. JAMA. 2014;311(22):2297-304. https://doi.org/10.1001/jama.2014.5988.

Publisher's Note Springer Nature remains neutral with regard to jurisdictional claims in published maps and institutional affiliations.

\section{Affiliations}

Alexander D. Miras ${ }^{1} \cdot$ Saranya Ravindra ${ }^{1} \cdot$ Anna Humphreys $^{1} \cdot$ Gerassimos Lascaratos $^{1} \cdot$ Kevin N. K. Quartey $^{1}$ (D $\cdot$ Ahmed Rashid Ahmed ${ }^{2} \cdot$ Jonathan Cousins ${ }^{2} \cdot$ Krishna Moorthy $^{2} \cdot$ Sanjay Purkayastha ${ }^{2} \cdot$ Sherif Hakky $^{2} \cdot$ Tricia Tan $^{1}$. Harvinder S. Chahal ${ }^{1}$

1 Section of Investigative Medicine, Division of Diabetes, Endocrinology \& Metabolism, Imperial College London, 6th floor Commonwealth Building, Hammersmith Hospital, Du Cane Road, London W12 0NN, UK
2 Department of Bariatric Surgery, Imperial College Healthcare NHS Trust, St Mary's Hospital, London W2 1NY, UK 